\title{
Utility Function-based Consumption Model under Continuous Time
}

\author{
Huawei Zhao
}

\author{
School of Mathematics, Sichuan University, Sichuan 610065, China
}

1549328983@qq.com

\begin{abstract}
The model of the portfolio distributing wealth to different assets in a certain proportion maximizes the expected utility function. The classic mean variance model proposed by Markowitz cannot meet the current demand for investment, while the dynamic portfolio model can more accurately describe the current changes in the price of different assets, which is more conducive to investors making corresponding decisions. The dynamic portfolio model not only provides a viable way for the inter-period distribution of limited resources, but also provides a guarantee for investors to predict the price of the market in the future. Therefore, it is of great significance to study the dynamic portfolio model. As a quantitative description of investor preference, the utility function is a direct way to measure the effectiveness of the portfolio model. Therefore, it is of great importance to study the portfolio model achieving the greatest expected utility. This paper mainly studies and improves the portfolio model based on CARA utility function and CRRA utility function. The nonparametric estimation and the method of solving differential equations are used to obtain the corresponding estimation expressions and first-order conditions. At the same time, the application of CRRA utility function in private equity is analyzed.
\end{abstract}

Keywords: portfolio model, CARA utility function, CRRA utility function.

\section{Introduction}

\subsection{Research Methods and Conclusions in Classical Literature}

With the continuous advancement of science and technology and the increasing development of production and management, the optimal consumption theory and method of utility functions has become an important theoretical basis and an indispensable method for evaluating consumer behavior and optimizing asset allocation, as evidenced by its wide application in all areas of economy and finance.

The utility function is to characterize the consumption behavior by relying on the function of quantity of goods so as to evaluate the related consumption behavior and obtain the optimal plan of assets allocation thus achieving the goal of revenue maximization. The theory of utility function has triggered long-term debates in history. Specifically, it was criticized mainly for the feature that it cannot be measured [1]. Then, the utility function was gradually replaced by the concept of preference. However, in the study of von Neumann and Morgan Stern, it is found that under certain reasonable assumptions, the utility function determined by the preference is one and only in the sense differed by one or similar translation, that is to say, under the uncertainty of probability, the utility function is measurable, and what people are pursuing is the maximization of the expected utility function.

According to their theory, when considering the general economic equilibrium without uncertainty, the behavior of economic activists is based on the maximized expectation of utility function. When taking into account of discussions of economic equilibrium with uncertainty, it is assumed that the quantities of commodities are all random variables, and their values will depend on the state of uncertainty. At this point, what we want is to compare the size of the utility after seeking mathematical expectations for such a utility function. That is, a utility function is directly defined on the set of random commodity quantities in question making it satisfies the expectation. The expectation of the function can be represented by a known function. A utility function that satisfies such condition is called a expected utility function. Von Neumann and Morgan Stern addressed the question of whether a utility function might be reasonably defined and existed. Since then, the utility function theory has been initially improved. 
The analysis of the utility function can help us effectively analyze the optimal asset allocation maximizing the benefits. When doing the analysis, it is often assumed that the economic activist we consider is the consumer, and the consumer has his own preferences for his activities. As the consumer activities can be characterized by the amount of goods consumed, yet with the uncertain environment, the events we need to select are all combinations of possible commodities. Therefore, consumers need to judge the pros and cons of all possible combinations.

Von Neumann has proposed the four famous axioms of the utility function.[1] When the commodity vector space we consider satisfies these four axioms, the utility function can be substantiated and measurable. This is critical for us to analyze consumer behaviors and develop strategies for the optimal asset allocation.

\subsection{Literature Review}

The utility function theory is mainly used in asset allocation throughout its history. Among them, the Markowitz portfolio model is the most widely used one. For the first time, his theory developed the ancient proverb "Don't put all the eggs in the same cage" to the theoretical level, and proposed specific solutions for people's portfolio behavior.

According to his theory, it is assumed that there are no different risk assets, their expected rate of return and their mutual benefit differences are known, then the "minimum variance" can be used as a condition to set the objective function, and the quadratic programming can be used to solve the issue of general mean variance portfolio based on the total expectation and corresponding expected linear rule.

The Lagrange multiplier method can be used to solve the portfolio model with the minimum risk.[2] The solution to the Markowitz model is the effective frontier research on the portfolio. A more indepth study was carried out by later generations on the basis of this theory. Specifically, based on the classic portfolio model of Markowitz, the existing utility functions were improved, and the properties of the utility function were analyzed by linear fitting. While at the same time, people are constantly exploring new utility functions to try to guide investors' behavior more accurately.

When the utility function is added, investors can measure their own preferences and risks with the help of the utility function, that is, the consumer's investment activities follow a utility function of gains and risk. In economics, the utility function refers to the indifference curve in the gain-standard deviation. After the utility function is determined, the two sides of the equation are separately differentiated to obtain the slope of the indifference curve.

In reality, investors often want to get high returns while avoiding risks as much as possible. When measured by utility function, it is the principle of utility maximization. The expected utility of an investor is a function of the expected return and risk of the portfolio of securities being invested. Different portfolio options correspond to different expected utility functions which develop different indifference curves in the yield-standard deviation plane in the form of parabola. On the same indifference curve, the yield and risk of the portfolio are consistent. Therefore, the asset allocation corresponding to these indifference curves and the cut-off point of the efficient frontier is the optimal strategy of asset allocation we need to find.

It can be found that it is highly effective to use the utility function theory to guide and analyze the asset allocation. But since it is quite difficult to establish the indifference curve of an investor, it is urgently needed to improve the utility function to make its corresponding indifference curve much easier to solve and fit the corresponding valid efficient frontier. In this paper, the CARA utility function and CRRA utility function are mainly selected for analysis to discuss their application in consumption, the deficiency of the portfolio model in the classic mode, thus accordingly improving the model.

\subsection{The Article is Divided into Seven Parts.}

The first part introduces the application of the classic literature and the current frontier literature on the utility functions in portfolio and consumer decision making. The second part introduces the relevant model of asset allocation under the specific CARA utility function and the application of the 
model in the corresponding asset allocation and consumption. The third part introduces the relevant models of asset allocation under the CRRA utility function, and analyzes the application of their corresponding models in durable goods consumption and non-durable goods consumption. The fourth part improves and optimizes the original CRRA model, and proposes a new CRRA model to analyze its application in consumption. The fifth part introduces the application of CARA utility function in American option pricing. The sixth part is a summary of the conclusions of this paper. The seventh section introduces the references cited.

\section{The Asset Allocation under the CARA Utility Function}

\subsection{Asset Allocation Model of CARA Utility Function}

Market Analysis and Model Configuration: It is assumed that there are $\mathrm{n}$ different risk assets and a risk-free asset in the market for investors to choose, and the yield vector of the known corresponding risk asset is $\theta=\left(\theta_{1}, \theta_{2}, \ldots . \theta_{n}\right)^{T}$. Set the risk-free rate to be $\mathbf{r}_{\mathrm{f}}$. The ratio vector invested in the risk portfolio is $\omega=\left(\omega_{1}, \omega_{2}, \ldots \ldots \omega_{\mathrm{n}}\right)^{T}:$ The proportion of investment in risk-free assets can be recorded as $\omega_{0}=1-\sum_{i=1}^{n} \omega_{i}$. Thus, the yield of the entire portfolio can be expressed as $\theta_{\mathrm{p}}=\omega_{0} \mathrm{r}_{\mathrm{f}}+\sum_{i=1}^{n} \omega_{\mathrm{i}} \theta_{i}$. Therefore, if we want to maximize the expected utility function, we can build model $\max _{\omega} E\left[U\left(\theta_{p}\right)\right]=E\left[U\left(\sum_{i=1}^{n} \omega_{i} \theta_{i}+\omega_{0} \boldsymbol{r}_{f}\right)\right]$.[3] Among them, $U()$ as the CARA utility function has the following form of $U(x)=-e^{-a x}, a>0, a$ is used to measure the degree of risk aversion.

Nonparametric Estimation of Models: In traditional portfolio models based on expected utility maximization, it is always assumed that asset returns are subject to a particular distribution, such as normal distribution, continuous-time Brownian motion, and so on. But in reality, it is difficult to know the information about the portfolio yield vector $\theta$. Therefore, we choose non-parametric estimates that require very low a priori information to predict and estimate the model's rate of return.

According to the corresponding econometric knowledge, we can get a non-parametric estimate of the density function $\mathrm{p}(\mathrm{x})$ of a random variable $\mathrm{X}$ is $p(x)=\frac{1}{T h} \sum_{i=1}^{T} K\left(\frac{X_{i}-x}{h}\right)$ after a given sample set of $\left\{X_{1}, \ldots \ldots X_{n}\right\}$, where $k()$ is a kernel function, $\mathrm{h}=\mathrm{h}(\mathrm{T})$ is a smooth function related to the sample size T called the window width. $k$ ( ) and T meets the following conditions:

$1 k()$ is non-negative and bounded, and there is $\int k(v) d v=1, k(-v)=k(v), \int v^{2} k(v) d v>0$

2 When meeting $T \rightarrow \infty$, then $h(T) \rightarrow 0$ and $T h(T) \rightarrow \infty$

Now suppose the sample set of the yield vector is $\left(R_{1}, R_{2} \cdots \cdots R_{n}\right)$, the corresponding investment decision is $\omega$,

Because $\theta=\omega_{0} \boldsymbol{r}_{f}+\sum_{i=1}^{n} \omega_{i} \theta_{i}=\omega^{T} \theta+\left(1-\omega^{T} I\right) r_{f}$

Then the combined rate of return is $\omega^{T} \theta=\left(\omega^{T} R_{1}, \ldots \cdots \omega^{T} R_{n}\right)$

It can be drawn that the non-parametric estimation of the density function is $p(x)=\frac{1}{T h} \sum_{i=1}^{T} k\left(\frac{\omega^{T} R_{i}-x}{h}\right)$ [4] 
It can be further drawn that the non-parametric estimation of $E\left[U\left(\omega^{T} \theta\right)\right]$ is $\int_{-\infty}^{\infty} U\left(x+\left(1-\omega^{T} I\right) r_{f}\right) p(x)$

It can be simplified as $\frac{1}{T h} \sum_{i=1}^{T} \int_{-\infty}^{+\infty} U\left(x+\left(1-\omega^{T} I\right) r_{f}\right) k\left(\frac{\omega^{T} R_{i}-x}{h}\right) d x$

Because the representation of CARA utility function is $U(x)=-e^{-a x}$, it can express $E\left[U\left(\omega^{T} \theta\right)\right]$ as

$$
\begin{aligned}
& E\left[U\left(\omega^{T} \theta+\left(1-\omega^{T} I\right) r_{f}\right)\right]=\frac{1}{T} \sum_{i=1}^{T} \int_{-\infty}^{+\infty} U\left(\omega^{T} R_{i}-z h+\left(1-\omega^{T} I\right) r_{f}\right) k(z) d z \\
& =-\frac{1}{T} \sum_{i=1}^{T} \int_{-\infty}^{+\infty} e^{-a\left(\omega^{T} R_{i}^{-z h+\left(1-\omega^{T} I\right)} r_{f}\right)} k(z) d z \\
& =-\frac{1}{T} e^{-a\left(1-\omega^{T} r_{f}\right)} \sum_{i=1}^{T} e^{-a} \omega^{T} R_{i} \int_{-\infty}^{+\infty} e^{a z h} k(z) d z
\end{aligned}
$$

and $z=\frac{\omega^{T} R_{i}-x}{h}$. Therefore, it only needs to select a specific $\mathrm{k}(\mathrm{x})$ to get the corresponding nonparametric estimate.[5]

\subsection{Application of CARA Utility Function in Durable Goods Consumption}

We only take into account of situations that can be made across time conditions. It is assumed that a kind of durable goods consumption has nothing to do with the expected cost of change; the difference between durable goods in different types of consumption is not considered; the ratio of service quality to stock is assumed to be equal to the wear rate of durable goods. Write the CARA utility function $U=U\left(x_{1}, \delta_{j} x_{2}\right), x_{1}$ is a non-durable goods consumption set, $x_{2}$ is a non-durable goods consumption set, $\delta_{j}$ is the corresponding wear rate. Since what satisfies the consumer's utility is only the service flow of the durable goods, $\delta_{j} x_{2}$ as one of the variables of a utility function also represents the ratio of the service flow to the stock.

The linear regression method can be used to write the CARA utility function as a linear form

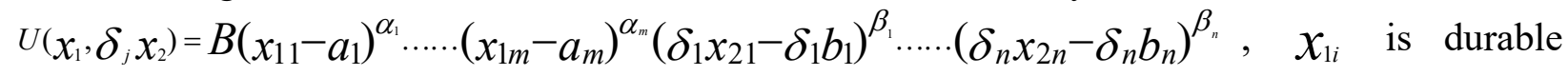
goods consumption, $x_{2 j}$ is non-durable goods consumption, $a_{i}$ is the minimum requirement for the $\mathrm{i}$-th non-durable goods consumption and $b_{j}$ is the minimum consumption of the $\mathrm{j}$-th durable goods consumption. $\alpha_{i}, \beta_{j}$ are constants whose size reflects the order of preference between different kinds of consumption. [6]

If the consumption is inter-temporal, that is, the consumer can get the utility of the service flow when using the durable goods, only paying the cost of the service flow with no need to pay the corresponding fee of remaining durable goods inventory. Then, the quantity of various consumer goods we buy under the condition of market price $\mathrm{P}$, according to the model

$$
\begin{aligned}
& \max U\left(x_{1 j}, \delta_{j} x_{2 j}\right) \\
& \text { s.t. } \sum_{i=1}^{m} p_{1 i} x_{i}+\sum_{j=1}^{n} \delta_{j} p_{2 j} x_{2 j}=M
\end{aligned}
$$

It can be obtained that 


$$
p_{2 j} x_{2 j}=p_{2 j} b_{j}+\frac{\beta_{j}}{\delta_{j}\left(\sum_{i=1}^{m} \alpha_{i}+\sum_{i=1}^{n} \beta_{i}\right)}\left[M-\left(\sum_{i=1}^{m} p_{1 i} a_{i}+\sum_{j=1}^{n} \delta_{j} p_{2 j} b_{j}\right)\right]
$$

indicating the $\mathrm{j}$-th durable goods consumption is a linear function of its price and income stock.

\subsection{Application of CARA Utility Function in Non-Durable Goods Consumption}

We still assume that the CARA utility function has the form of $U(x)=-e^{-a x}$ with $a>0$ representing the degree of risk aversion. When analyzing consumer behavior and decision making, it is usually assumed that the consumer is completely rational meaning that consumers are always able to choose a combination of high-yield and low-risk assets in the various affordable consumer combinations that are available, that is to say, a portfolio can always maximize its expected utility. In the traditional research, it is assumed that the consumer's preference relationship is continuous; therefore, durable goods consumption can be regarded as one-time non-durable goods consumption.

Because the consumer can only purchase once after making multiple savings, the following model can be built.

$$
\begin{aligned}
& \max U\left(x_{1 i}, x_{2 i}^{\prime}\right) \\
& \text { s.t. } \sum_{i=1}^{m} p_{1 i} x_{1 i}+\sum_{j=1}^{n} p_{2 j} x_{2 j}=M^{\prime}
\end{aligned}
$$

While $x_{1 i}$ is non-durable goods consumption, $x_{2 j}^{\prime}$ is durable goods consumption, $M^{\prime}=b W_{t}^{,}$and $W_{t}^{\prime}=A_{t-1}\left(1+r_{t}\right)+Y_{\theta}$ represents disposable wealth. A is the stock of actual financial assets and $Y_{\theta}$ is the income of human capital.

It can be obtained that

$$
p_{2 j} x_{2 j}^{\prime}=p_{2 j} b_{j}+\frac{\beta_{j}}{\sum_{i=1}^{m} \alpha_{i}+\sum_{h=1}^{n} \beta_{h}}\left[M^{\prime}-\left(\sum_{i=1}^{m} p_{1 i} a_{i}+\sum_{j=1}^{n} p_{2 j} b_{j}\right)\right] \text { is the consumer demand }
$$

function of the consumer in the non-durable goods consumption behavior of only one-time purchase. Since the no cross-time consumption is considered, the non-durable goods consumption at this time can also be regarded as durable goods consumption.

\section{The Asset Allocation Model under CRRA Utility Function and its Improvement}

\subsection{Current State of Research on CRRA Utility Function Portfolio Model}

How to maximize utility is one of the most basic problems in the field of research on asset pricing theory, and also the cornerstone of economic finance.[7] It has experienced rapid development in this field since Markowitz proposed the mean variance model of capital assets.[1]

The dynamic portfolio theory based on the expected utility function theory is a stochastic dominance criterion based on the Von Neumann utility function. Because this theory is in line with investors' expectations, it is widely accepted in the uncertainty decision theory. Later, many scholars extended this theory in different directions. For example, Merton studied how to use the multi-random factor model to describe the dynamic process of asset price under the assumption of continuous time and use dynamic programming to solve the optimal consumption and portfolio decisions based on the maximized expected utility.[8] Liu and Pan introduced the jump process in the stochastic volatility model, and used the power utility function as the hypothetical utility function of the investor, thus 
obtaining the approximate solution of the portfolio selection.[9] Under the CRRA utility function, Brandt and Santa introduced predictor variable to expand the asset space, forming a manageable portfolio, and obtained the optimal allocation of risk assets in the dynamic portfolio through the simulation of state variables. Lorenzo Garlappi developed the utility function through Taylor's formula and used it as a linear approximation to solve the dynamic portfolio problem. This method greatly improved the accuracy of the result. For the first time, the approximate values of expected utility function given by the condition information was proposed, pointing out that under the assumption that the return on assets is biased, the income distribution can be approximated by Taylor expansion, thereby improving the accuracy and efficiency of the portfolio. Marcel Prokopczuk conducted an empirical data analysis on the dynamic portfolio under system risk constraints to solve the dynamic portfolio problem of the CRRA utility function under the assumption that the asset investment price obeys the Possion jump process. It is concluded that a portfolio that ignores systemic risk will reduce expected returns and expected utility.[10]

The research on asset portfolio is relatively rare and relatively imperfect under the expected utility function. Zhang Wei and Bai Xiaohong proposed to replace the indifference curve with an investment preference curve to study the drift trajectory and properties of the portfolio curve when the market environment was in a consistent expected change. Guo Lei and Chen Fang constructed a continuous dynamic programming model based on the preference of CRRA utility function, and obtained the optimal individual investment strategy for enterprise annuity before and after retirement. Yuan Zijia and Li Zhongfei studied how to influence the risk caused by parameter uncertainty in the standard investment theory, which will affect the investment decision, and use the martingale method to derive the expression formula of the optimal strategy of the CRRA utility function.

\subsection{Asset Allocation and Model of CRRA Utility Function}

When analyzing the constant relative risk aversion utility function, the following assumptions are required in the past classical model, expressed as:

First, the preference function is only related to the final wealth;

Second, the preference relationship is complete, transitive and consistent;

Third, when formulating investment strategies, the estimation of the level of preference for each strategy is expressed by the probability of occurrence of the result;

Fourth, utility is only related to the final result, and has nothing to do with the process of achieving the result.

With the above assumptions, the CRRA utility function can be expressed as the following form

$$
U(W)=\frac{W^{1-\gamma}}{1-\gamma}, \quad(\gamma>1)
$$

$\gamma$ represents the relative risk aversion coefficient used to measure the risk aversion of investors [11]

In the past, the investment model of CRRA utility function was mainly based on BS model, and its basic form was:

$$
\frac{d S_{t}}{S_{t}}=u d t+\sigma d Z_{t}
$$

$S_{\mathrm{t}}$ is the asset price, $Z_{t}$ is the standard Brownian motion. The basic assumptions of the BS model are as follows:

First, the stock price obeys a lognormal distribution, and its expected return and variance are constant;

Second, the stock does not pay dividends;

Third, there is no handling fee for stocks and options trading.

Since these assumptions are difficult to achieve in reality, later generations have made some improvements to the BS model and introduced the jumping process. The specific form is as follows 


$$
\frac{d S_{t}}{S_{t}}=u d t+\sigma d z_{t}+x_{t} d N_{t}
$$

$x_{t}$ indicates the degree of jump fluctuations in stock prices due to the occurrence of various large events, and obeys a lognormal distribution. $N_{t}$ is the Poisson process of strength. This improved BS model uses the differential equations of the Poisson process and the Brownian motion to analyze the fluctuation process of stock prices to guide investors in making corresponding decisions and thus gaining profits. However, this improved model still has some shortcomings. For example, the introduction of jump volatility $\mathcal{X}_{t}$ has the assumption of obeying the lognormal distribution, and it cannot reflect the bias of the yield distribution.

\subsection{Application and Improvement of CRRA Utility Function Model}

The application of the CRRA utility function investment model is mainly reflected in the net present value model and the random walk model. Among the models involved in the rational hypothesis are Williams' net present value model and Osborn's random walk of stock prices.[12] The former assumes that investors can judge the value of securities and take corresponding decisions through rational expectations of the future earnings and expected returns of securities. The trading behavior of investors depends on the basic information of securities. The latter argues that stock prices follow the theory of random walks and believes that investors value stocks based on their expected value or rate of return. While the expected value is the probability-weighted average of the possible yields, the investor rationality defined by Osborn is the subjective probability set in an unbiased manner.

The issue of asset allocation is considered under the assumptions of the above CRRA utility function: set the investor's original wealth as $W_{0}$, in the consideration of the income of a risky asset and a risk-free asset (ie bond). The fixed income of the bond is $r$, and an uncertain return produced by the risky asset is $\gamma$, both of which are calculated as continuous compound interest. Investors choose a portion of the original assets $\alpha$ to invest in the risk assets to maximize the expectations of the end-of-life wealth $W$. Then the asset allocation can be expressed as

$\max E[U(W)]$

and $W=\alpha W_{0}\left[e^{\gamma}-e^{r}\right]+W_{0} e^{r}$

expressing in the form of the utility function of CRRA as $U(W)=\frac{W^{1-\gamma}}{1-\gamma}$

Later, it was found that if this model is used to explain the issue of stock premium, it requires very strict conditions. For instance, it is necessary to ensure that investors have a very strong risk aversion, yet such explanation often has a large deviation from the actual situation. Therefore, it is urgently needed for a more realistic model to explain such issue. The loss aversion model is the most mature one in many improved models.

In the loss aversion model, it is assumed that people always consider firstly how to avoid losses, and then how to maximize the gains. And the value function $\mathrm{v}$ is used to replace the utility function in the traditional model, and the decision weight function $\pi(p)$ used to replace the probability $p$ of expected utility function.

The value function in this model enjoys the following features:

First, the value function is a curve about the monotonous increase of yield, because people always prefer income and hate losses, suggesting that the higher the return is, the greater the corresponding value is.

Second, the independent variables in the value function are related to the change of the reference point. If the selected reference points are different, the independent variables are also different. If there is no gain or loss, the value function is 0 . Therefore, when the reference point is selected, the 
reference point is taken as the origin, the gain and loss as the coordinate axes, then the value function is a monotonically increasing curve passing the origin.

Third, the s-type based value function is centered on the origin. When the return is positive, the value function is up convex, and when the return is negative, the value function is down convex.

This theory is also called the outlook theory. The outlook theory mainly includes the following points: First, what affects investors' decision-making is the change of wealth rather than the final value; Second, investors tend to take risks when facing considerable losses and tend to accept certain yields when facing some favorable conditions; Third, the behavior of the agent is not unchanged; Fourth, the happiness brought by yield and the pain caused by the same loss are not equal. The latter is greater than the former and so on. This theory formally proposed by Kahneman and Tversky in 1979 divided the investor's decision process and selection process into two stages according to the value function and the decision weight function, and provided the investment decision model for the investors under uncertain conditions in this regard.

\section{The Frontier Application and Analysis of CRRA Model}

\subsection{Portfolio Model with Jump Price}

What we are considering is whether, under the established effective market hypothesis, the information already available to investors can be used to make an unbiased estimation of the CRRA utility function, so as to obtain a corresponding investment strategy and obtain considerable yields.[13] For doing so, we need to assume that the efficient market hypothesis is established and that the prices of the different assets are sufficient to reflect all of the public information it corresponds to which includes all information that has been disclosed or not yet disclosed, such as transaction price, volume, company financial management information, profitability, profit forecast value, and so on.

The requirements for the establishment of the effective market hypothesis are as follows: First of all, each and every investor is a rational investor who conducts an analysis of the corresponding asset price at all times in order to seek a balance between gain and risk; secondly, the price of assets reflects people's expectations of the future price of such assets, thus achieving a balance between supply and demand in the capital market. Otherwise, there will be arbitrage opportunities in the capital market; thirdly, the price changes of a commodity fully reflect the information that changed contained in it meaning that the related positive or negative news will be immediately reflected in the corresponding changes in its price. Therefore, the "Efficient Market Hypothesis" ensures that there is no arbitrage opportunity in an efficient market, that is, "there is no free lunch in the world".

Therefore, we should determine the following conditions when establishing the corresponding investment model:

First, investors have sufficient funds and there are a sufficient number of investors in the market, and each of them can purchase any asset in the market at will.

Second, the assets in the market can be distributed with absent of the cost of the transaction and the corresponding tax.

Third, the investor's risk utility function is the CRRA utility function.

Fourth, allow investors to trade all capital and allow all assets to be sold short.

Fifth, the investor's transaction is conducted under the model of continuous time.

Sixth, the capital market is always in equilibrium and there are no transactions with unbalanced prices.

Seventh, risk assets and risk-free assets existing in the capital market are always subject to the yield jump model.

Based on these conditions, we can build a dynamic portfolio model for risk-free assets and risk assets in continuous time:

$$
\frac{d S_{i}}{S_{i}}=r d t(i=0,1, \ldots \ldots n)
$$


When there is a jump, the corresponding model is

$$
\frac{d S_{i}}{S_{i}}=\left(r+\alpha_{i}\right) d t+\sigma_{i} d z_{i}+x_{i} d N_{i}(\lambda) \quad(i=0 \ldots \ldots N)
$$

$\mathrm{r}$ represents the fixed rate of return on risk-free assets, $\alpha_{t}$ represents the excess return of the asset, $\sigma_{\mathrm{t}}$ indicates the volatility of the corresponding investment asset, $d Z_{t}$ represents the ndimensional standard Winner process, $N_{\mathrm{i}}(\lambda)$ indicates the standard Poisson distribution of the parameter, $X_{t}$ is the jump in the random jump amplitude.[14]

If taking $\omega_{\mathrm{i}}(\mathrm{i}=1 \ldots \mathrm{n})$ indicating the proportion of the investment shares of the corresponding $\mathrm{n}$ types of risk assets, the above model of the jump can be changed to:

$$
d W_{t}=W_{t}\left[\left(r+\omega_{t}\right) d t+\omega_{t} \sigma d z_{t}+\omega_{t} X_{t} d N_{t}(\lambda)\right]
$$

$W_{\mathrm{t}}$ represents the investor's capital amount.

As an investor, what we want is to maximize the utility by determining the asset allocation (the CRRA utility function is used here), then $\max E\left[U\left(W_{t}\right)\right]$

Now solve this, make $J\left(W_{t}, t\right)=\max E\left[U\left(W_{t}\right)\right]$, then $J\left(W_{t}, t\right)=b(t) \frac{W_{t}^{1-\gamma}}{1-\gamma}$. If there is an optimal solution to this, then there is $\frac{\partial J}{\partial W}=0, \frac{\partial J}{\partial \mathrm{t}}=0$. Take the expected and substitute

$$
0=\max \left\{\frac{\partial J}{\partial t}+\frac{\partial J}{\partial W_{t}}\left[r W_{t}+\omega_{t} \alpha W_{t}\right]+\frac{1}{2} \frac{\partial^{2} J}{\partial W^{2}} W_{t}^{2} \omega_{t}^{T} \Sigma \omega_{t}\right.
$$

Because $\quad J\left(W_{t}, t\right)=b(t) \frac{W_{t}^{1-\gamma}}{1-\gamma}$, then $\frac{\partial J}{\partial t}=b^{\prime}(t) \frac{W_{t}^{1-\gamma}}{1-\gamma}, \quad \frac{\partial J}{\partial W}=b(t) W_{t}^{-\gamma}$, $\frac{\partial^{2} J}{\partial W^{2}}=-b(t) \gamma W^{-1-\gamma}$

Substitute it

Simplified it

$$
\begin{aligned}
& 0=\max \left\{b^{\prime}(t) \frac{W_{t}{ }^{1-\gamma}}{1-\gamma}+b(t) W_{t}{ }^{-\gamma} W_{t}\left[r+\omega_{t} \alpha\right]-\frac{1}{2} b(t) \gamma W_{t}{ }^{-1-\gamma} W_{t}{ }^{2} \omega_{t}{ }^{T} \sum \omega_{t}\right. \\
& \left.+\lambda E\left[b(t) \frac{\left(W_{t}+W_{t} \omega_{t}{ }^{T} X_{t}\right)^{1-\gamma}}{1-\gamma}-b(t) \frac{\omega_{t}{ }^{1-\gamma}}{1-\gamma}\right]\right\}
\end{aligned}
$$

$0=\alpha-\gamma \sum \omega_{t}+\lambda E\left[X_{t}\left(1+\omega^{T} X_{t}\right)^{-\gamma}\right]$ is the first order condition

It can be drawn that: in the selection of dynamic portfolio with Poisson jump process, when meeting the above formula $d W_{t}=W_{t}\left[\left(r+\omega_{t}\right) d t+\omega_{t} \sigma d z_{t}+\omega_{t} X_{t} d N_{t}(\lambda)\right]$ and the constraints of the CRRA utility function, the maximization of the expected utility can only be achieved in corresponding asset allocation after satisfying the above first-order condition. 


\subsection{Portfolio Model under Delayed Investment [15]}

Here we mainly consider the issue of investment with private equity. At present, the research of this issue in the academic frontier mainly considers the problem of maximizing the utility of investment under the assumption of rational investors, that is, the CRRA utility function as the investment utility of maximizing utility under the expected utility function. In this model, the wealth expectation of private equity investment in the case of delay and no delay is equal as the opportunity cost, and the corresponding mathematical cost is used to find the condition that minimizes the opportunity cost to determine the maximum return rate of the portfolio. [16].

While the standard deviations of the private equity fund yield and the risk stock's rate of return are represented respectively as $\sigma_{p} 、 \sigma_{s}$, their respective Brownian movements as $Z_{t}[17]$.

Under the relevant continuous hypothesis, [18] we can define its weight in the portfolio as follows

$$
\begin{aligned}
& x=\frac{C_{t}}{W_{t}} \\
& s=\frac{S_{t}}{W_{t}} \\
& b=\frac{B_{t}}{W_{t}}
\end{aligned}
$$

and $W_{\mathrm{t}}=C_{t}+S_{t}+B_{t}$

For the sake of convenience, we consider the case where the investor invests in two stages. At the initial moment (recorded as time 0), the investor invests part of the entire capital into the private equity fund, and the rest of the funds are invested in the second phase. Write $\tau_{1}=\left[0, t_{1}\right]$, $\tau_{2}=\left[t_{1}, T\right]$, then it's easy to know $\tau_{1}$ and $\tau_{2}$ is the time to stop, so there is

$$
\begin{gathered}
\frac{d W_{t}}{W_{t}}=\left(\delta u_{p}+(1-\delta) u_{s}\right) d t+\delta \sigma_{p} d Z_{p, t}+(1-\delta) \sigma_{s} d Z_{s, t}, \text { when } t \in \tau_{1} \\
\frac{d W_{t}}{W_{t}}=u_{p} d t+\sigma_{p} d Z_{p, t} \text { when } t \in \tau_{2}
\end{gathered}
$$

If $\theta$ indicates the proportion of investment stocks, then:

When $t \in \tau_{1}$

$$
\frac{d W_{t}}{W_{t}}=\left(\delta u_{p}+(1-\delta)\left((1-\theta) r+\theta u_{s}\right)\right) d t+\delta \sigma_{p} d Z_{p, t}+(1-\delta) \theta \sigma_{s} d Z_{s, t}
$$

When $t \in \tau_{2}$ then $\frac{d W_{t}}{W_{t}}=u_{p} d t+\sigma_{p} d Z_{p, t}[19]$

Therefore, according to Ito Lemma, 


$$
d \ln W_{t}=\frac{d W_{t}}{W_{t}}-\frac{1}{2}\left(\frac{d W_{t}}{W_{t}}\right)^{2}
$$

When $t \in \tau_{1},=\left(\delta u_{\mathrm{p}}-\frac{1}{2} \delta^{2} \sigma_{p}^{2}+(1-\delta)\left((1-\theta) r+\theta u_{s}\right)-\frac{1}{2}(1-\delta)^{2} \theta^{2} \sigma_{s}^{2}\right.$

$$
\left.-\delta(1-\delta) \theta \sigma_{p} \sigma_{s} \rho\right) d t+\delta \sigma_{p} d Z_{p, t}+(1-\delta) \theta \sigma_{s} d Z_{s, t}
$$

$\rho$ represents the correlation coefficient between private equity and risk stocks After substituting the expression, the following results are:

$$
\begin{aligned}
& \ln W_{t_{1}}=\left(\delta u_{p}-\frac{1}{2} \delta^{2} \sigma_{p}^{2}+(1-\delta) u_{s}-\frac{1}{2}(1-\delta)^{2} \sigma_{s}^{2}-\delta(1-\delta) \sigma_{p} \sigma_{s} \rho\right) t_{1} \\
& +\delta \sigma_{p} d Z_{p, t_{1}}+(1-\delta) \sigma_{s} d Z_{s, t_{1}} \\
& \ln W_{T}-\ln W_{t_{1}}=\left(u_{p}-\frac{1}{2} \sigma_{p}^{2}\right)\left(T-t_{1}\right)+\sigma_{p}\left(Z_{p, T}-Z_{p, t_{1}}\right) \\
& \ln W_{T}=\left(u_{p}-\frac{1}{2} \sigma_{p}^{2}\right)\left(T-t_{1}\right)+\left(\sigma u_{p}-\frac{1}{2} \delta^{2} \sigma_{p}^{2}+(1-\delta) u_{s}-\frac{1}{2}(1-\delta)^{2} \sigma_{s}^{2}\right. \\
& \left.-\delta(1-\delta) \sigma_{p} \sigma_{s} \rho\right) t_{1}+\delta \sigma_{p} d Z_{p, t_{1}}+(1-\delta) \sigma_{s} d Z_{s, t_{1}}+\sigma_{p}\left(Z_{p, T}-Z_{p, t_{1}}\right)
\end{aligned}
$$

Therefore, the utility function of the ultimate wealth of the investment can be obtained after expectation:

$$
\begin{aligned}
& E\left[u\left(W_{T}\right)\right]=\frac{1}{1-\theta} \exp \left[(1-\theta)\left(u_{p}-\frac{1}{2} \sigma_{p}^{2}\right) T+(1-\theta)\left(\delta u_{p}-u_{p}-\frac{1}{2} \delta^{2} \sigma_{p}^{2}\right.\right. \\
& \left.\left.+\frac{1}{2} \sigma_{p}^{2}+(1-\delta) u_{s}-\frac{1}{2}(1-\delta)^{2} \sigma_{s}^{2}-\delta(1-\delta) \sigma_{s} \sigma_{p} \rho\right) t_{t}\right] \\
& E\left[\exp \left[(1-\theta)\left(\delta \sigma_{p} Z_{p, t_{1}}+(1-\delta) \sigma_{s} Z_{s, t_{1}}+\sigma_{p}\left(Z_{p, T}-Z_{p, t_{1}}\right)\right)\right]\right]
\end{aligned}
$$

Since $\mathrm{Z}$ is a Brownian motion, it can be drawn by calculating the above formula:

$$
\begin{aligned}
& E\left[u\left(W_{T}\right)\right]=\frac{1}{1-\theta} \exp \left[(1-\theta)\left(u_{p}-\frac{1}{2} \sigma_{p}^{2}\right) T-(1-\theta)(1-\delta)\left(u_{p}-u_{s}-\frac{1}{2}(1+\delta) \sigma_{p}^{2}\right.\right. \\
& \left.+\frac{1}{2}(1-\delta) \sigma_{s}^{2}+\delta \sigma_{p} \sigma_{s} \rho\right) t_{1}+\frac{1}{2}(1-\theta)^{2}\left(\sigma_{p}^{2}\left(T-t_{1}\right)+\delta^{2} \sigma_{p}^{2} t_{1}\right. \\
& \left.\left.+(1-\delta)^{2} \sigma_{s}^{2} t_{1}+2 \delta(1-\delta) \sigma_{p} \sigma_{s} \rho t_{1}\right)\right]
\end{aligned}
$$

It can be concluded that the expression of the opportunity cost $\mathrm{p}$ in the case of delayed investment is:

$$
p=\frac{(1-\delta) t_{1}}{T}\left[u_{p}-u_{s}-\frac{1}{2} \theta(1+\delta) \sigma_{p}^{2}+\frac{1}{2}(1-\delta) \sigma_{s}^{2}+\theta \delta \sigma_{p} \sigma_{s} \rho\right]
$$




\section{Application of CARA Utility Function in Option Pricing}

The issue of option pricing is essentially important in the research of financial mathematics. We consider the issue of option pricing of option holders under the CARA utility function. It is hoped that the general pricing formula of American options can be obtained according to the Black-Scholes option pricing formula and the optimal stopping theory.

Suppose $\left(\Omega, F_{\mathrm{n}}, F, P\right)$ is a complete filter subspace, $\left\{S_{n}\right\}_{0 \leq n \leq t}$ is the Markov chain of this space. If the stock price $S_{n}$ satisfying $S_{n}=\left(1+\rho_{n}\right) S_{n-1}$ and $S_{0}=\mathrm{s}$, the stock price can be expressed as $S_{\mathrm{n}}=\left(1+\rho_{n}\right)\left(1+\rho_{n-1}\right) \ldots \ldots\left(1+\rho_{1}\right) \mathrm{s}$. We assume $\left\{\rho_{\mathrm{n}}\right\}$ as a sequence of independent and identically distributed random variables and can only take two values $a, b$, where $0<a<r<b$ and $\mathrm{r}$ is the risk-free rate. $Q$ is the probability measure subspace of $P$, satisfying.

$$
Q\left(\rho_{1}=a\right)=\frac{b-r}{b-a}=\bar{p}, Q\left(\rho_{1}=b\right)=\frac{r-a}{b-a}=\bar{q} \cdot[21]
$$

If $\forall 0<n<T$ and satisfying $E\left[X_{n}\right]<\infty$, define $\gamma_{n}=\max _{\tau \in T_{n}} E\left[X_{\tau} \mid F_{n}\right]$ as the sequence $\left\{X_{n}\right\}$ of Snell envelopes. According to the backward circulation property of the Snell envelope, $\gamma_{\mathrm{T}-1}=\max \left\{X_{T-1}, E\left[\gamma_{T} \mid F_{T}\right]\right\}$ the simplify it as:

$$
\gamma_{T-1}=\max \left\{\frac{-\exp \left(-\lambda S_{T-1}\right)}{(1+r)(T-1)}, E\left[\frac{-\exp \left(-\lambda\left(1+\rho_{t}\right) S_{T-1}\right)}{(1+r)^{T}} \mid F_{T}\right]\right\}
$$

Because $E\left[\frac{-\exp \left(-\lambda\left(1+\rho_{t}\right) S_{T-1}\right)}{(1+r)^{T}} \mid F_{T}\right]=\frac{1}{(1+r)^{T}} E\left[-\exp \left(-\lambda\left(1+\rho_{t}\right) S_{T-1}\right)\right] \leq \frac{-\exp \left(-\lambda S_{T-1}\right)}{(1+r)^{T-1}}$

Then $\gamma_{T-1}=\frac{-\exp \left(-\lambda \mathrm{S}_{\mathrm{T}-1}\right)}{(1+r)^{T-1}}=X_{T-1}$. Then it can be found by recursion, if any $\mathrm{m}$ satisfied $n \leq m \leq T-1$, then there is $\gamma_{m}=\frac{-\exp \left(-\lambda S_{m}\right)}{(1+r)^{m}}$

Now write $D=\{x: \bar{p} U[(1+b) x]+\bar{q} U[(1+a) x] \leq(1+r) U(x)\}$, then $U(x)=-e^{-\lambda x}$ is the CARA utility function, then D can be written as $[d, \infty)$, because $S_{n-1} \notin D$, then there is

$$
\gamma_{n-1}=\max \left\{X_{n-1}, E\left[\gamma_{n} \mid F_{n-1}\right]\right\}=\max \left\{X_{n-1}, E\left[X_{n} \mid F_{n-1}\right]\right\}=\max \left\{X_{n-1},(1-r)^{-n} E\left[-\exp \left(-\lambda S_{n}\right) \mid F_{n-1}\right]\right\}
$$

Substitute $S_{n}$ and we can know that $\gamma_{n-1}>\frac{-\exp \left(-\lambda S_{n-1}\right)}{(1+r)^{n-1}}$ so the option pricing can be expressed as $C^{*}=E^{*}\left[\frac{-\exp \left(-\lambda S_{\tau^{*}}\right)}{(1+r)^{\tau^{*}}}\right], \tau^{*}$ is the optimal stop time and can be expressed as:

$\left\{\tau^{*}=n\right\}$, extract $\mathrm{i} \quad(1+\mathrm{b})$ from $(1+\mathrm{P} 1)(1+\mathrm{P} 2) \ldots \ldots(1+\mathrm{Pn})$ and calculate the available American option pricing expression as:

$$
C^{*}=\sum_{n=I}^{T} \sum_{i=I}^{T} C_{N}^{i} \bar{p}^{i} q^{-\overline{n-i}} \frac{-\exp \left(-\lambda S(1+b)^{i}(1+a)^{n-i}\right.}{(1+r)^{n}}
$$




\section{Conclusion}

This paper mainly discusses how to maximize the expected utility of the utility function in order to find out the corresponding first-order conditions in different situations to guide investors to make strategies in the allocation of investment. The choice of utility function mainly based on CARA utility function and CRRA utility function. The two utility functions are analyzed successively to obtain and the application of the two in consumption and investment portfolio, and improve the CRRA utility function accordingly making in-depth study of its application in the portfolio model. This paper has achieved the following results:

(1) For the portfolio model under the CARA utility function, the non-parametric estimation method can be used to obtain the non-parametric estimation expression of the expected utility, simplifying the portfolio problem model. After that, this paper analyzes the application of CARA utility function in durable goods consumption and non-durable goods consumption, and draws the conclusion that the consumption of durable goods is a linear function of its price and income stock. A consumer demand function for non-durable goods consumption that has been purchased once has been derived. (2) For the portfolio model under the CRRA utility function, this paper obtains a new portfolio model form after introducing the Poisson jump process, and compares the defects of the new model in the stock premium, and gets more efficient loss aversion models and related features. (3) The portfolio model with jump interval under the CRRA utility function is established, and the corresponding firstorder condition for maximizing the expected utility is obtained. The CRRA utility function is applied to the private equity investment with delayed investment. The minimum opportunity cost is used to solve the corresponding differential equations, thus obtaining the final opportunity cost expression. (4) Under the assumption of the CARA utility function, the general form of the American option pricing formula is derived based on the general Black-Scholes formula.

Improvement of the model: Based on the CARA utility function and the CRRA utility function, this paper makes a certain in-depth study on the risk portfolio model, yet there are still some problems that need to be further studied and discussed. In particular, several directions are proposed below: First, this paper based on Von Neumann's classic utility function portfolio theory and the efficient market hypothesis mainly theoretically solves and analyzes the portfolio of CARA and CRRA utility functions with the establishment of the corresponding assumptions for the model. Then how to optimize these necessary assumptions is for further exploration. Secondly, this paper mainly studies the theoretical part and solves the expression of the corresponding model without collecting the actual data to evaluate the validity of the model hinting that The detection of the actual data needs to be improved.

Prospects for further research: the research on the study of dynamic portfolios by foreign scholars has been relatively mature, while the domestic research is in its infancy, and most of them are based on theoretical research with few practical applications. Therefore, there is still much room for improvement in the study of dynamic portfolios. This paper, based on the CARA and CRRA utility functions, theoretically analyzes the portfolio model filling the gaps in domestic research to a certain extent. The research results suggest that the portfolio model and the first-order conditions obtained by different utility functions are different, and different utility functions often depend on the risk aversion level of different investors. Therefore, whether it can be more objectively and accurately described the level of risk aversion of investors will largely determine the accuracy of the utility function. Accurately and quantitatively describing the risk aversion of different investors help to develop a portfolio model that suits different investors, which will be the new direction of research on the portfolio strategy in the future.

\section{References}

[1]. Shi Shuzhong. Ten Lectures on Financial Economics [M]. Shanghai People's Publishing House, 2011. 66-134. 
[2]. Gao Tiemei. Econometric Analysis Method and Modeling [M]. 2nd ed. Tsinghua University Press, 2009.3-238.

[3]. Yao Haiyang. Portfolio Selection Based on Non-parametric Estimation Method and CARA Utility Function [A]. Proceedings of the 13th China Management Science Academic Conference, 2011.

[4]. Robert, C. 1970, "Optimum consumption and portfolio rules in a continuous-time model", Journal of Economic Theory, Vol.3, PP373-402.

[5]. Lo, A.W., 1986, "Maximum likelihood estimation of generalized ito processes with discretely sampled data", Economical Theory, Vol.4, PP231-240.

[6]. Shao Jianping, Chen Tibiao. Analysis of Consumption Models Containing Durable Goods[J]. Journal of Lanzhou University of Commerce, 2002, 18(6): 27-29.

[7]. He Chaolin. Research on Dynamic Asset Portfolio Selection under Continuous Time [D]. PhD thesis of Chongqing University, 2007.

[8]. Merton. R.C. 1971, "Optimum consumption and portfolio rules in a continues-time model", Journal of Economic Theory, Vol.4, PP373-413.

[9]. Liu. J. \&Pan. J., 2003, “Dynamic derivative strategies”, Journal of Financial Economics, Vol.69, PP401-430.

[10]. Marcel Prokopczuk, 2011, "Optimal portfolio choice in the presence of domestic systemic risk: empirical evidence from stock markets", Decisions Economic Financial, Vol.34, PP141168.

[11]. Gul Faruk.1991, “A theory of disappointment aversion”, Econometrical, Vol.59, PP667-690.

[12]. Li Zhongfei, Mei Lin. Comparative Analysis of Three Utility Models of CRRA, LA and DA-The Evolution and Development of Asset Allocation Theory [J]. Management Review,2004, (11):11-27.

[13]. Lin Yuanlie. Application of Stochastic Process [M]. Tsinghua University Press, 2002.

[14]. Gao Qing. Empirical Analysis of Dynamic Portfolio Model Based on CRRA Utility Function [D]. Master's Thesis of Dongbei University of Finance and Economics, 2014. (3): 14-28.

[15]. Merton, R.C.,1973, “An intertemporal capital asset pricing model”, Econometrical, Vol.41, PP867-887.

[16]. Yang Liu \& Jingqiang Yang. 2015, "Optimal investment of private equity", Finance Research Letters, Vol. 14, PP76-86.

[17]. Wang Jiao. Continuous Time Optimal Consumption and Portfolio with Private Equity [D]. Master's Thesis of Guizhou University, 2017.

[18]. Merton, R. C,1992, “Continuous-Time Finance”, Wiley-Blackwell, Vol.27, PP13-127.

[19]. Beckers, S. 1981, "A note on estimating the parameters of the diffusion-jump model of stock return", Journal of Financial and Quantitative Analysis, Vol.16, PP125-140.

[20]. Kim. T. S. \& E. Omberg, 1996, "Dynamic derivative no myopic portfolio behaviour”, The review of Financial Studies, Vol.69, PP401-430.

[21]. Xing Yingchun. Pricing of American Options Under the CARA Utility Function, 2011, (28): $18-20$. 